\title{
Lehrer, Professoren und Studenten in der 250-jährigen Geschichte der TU Bergakademie Freiberg
}

\author{
von \\ HARTMUT SCHLEIFF
}

Die Institutionengeschichte der Bergakademie Freiberg seit ihrer Gründung im Jahr 1765 aus der sie formenden Lehrer- und Professorenschaft sowie den Studierenden herauszuarbeiten, heißt über verschiedene Staatsordnungen hinweg zu vergleichen. Erstmals werden im vorliegenden Beitrag die 535 Biografien der Lehrer beziehungsweise Professoren aus den ersten 250 Jahren der ältesten technischen Bildungsakademie Sachsens zugrunde gelegt, um folgende Gesichtspunkte statistisch zu diskutieren.

Zum einen ist der Fächerkanon, der die Bergakademie Freiberg über die verschiedenen Staatsordnungen hinweg prägte, aufzuzeigen, steht doch die Bergakademie insbesondere für Forschung und Lehre zur Rohstoffgewinnung und Rohstoffverarbeitung sowie damit korrespondierende Fächer. Um die Gewichtung der Fächer in der Entwicklung der Bergakademie und damit disziplinäre Schwerpunktsetzungen verdeutlichen zu können, sind drei Fachbereiche analytisch zusammengefasst worden: erstens die Naturwissenschaften mit der Mathematik, dann die Wirtschafts-, Rechts-, Verwaltungs- und Geisteswissenschaften sowie drittens die Technikwissenschaften.

Ein weiterer Gesichtspunkt sind die akademischen Lebensverläufe der Professoren mit ihren jeweiligen Qualifikationsabschlüssen und den von ihnen durchlaufenen Bildungskarriereinstitutionen. Sie geben einen Einblick in den Wirkungsradius der Bergakademie Freiberg in Wissenschaft, Wirtschaft und Politik. Insbesondere ist das Verhältnis der Freiberger Professoren zu Wirtschaft und Politik herauszustellen, da die Bergakademie als höhere technische Bildungsanstalt des sächsischen Bergstaates gegründet wurde, also als Teil der Verwaltung Sachsens. Für die Zeit nach Auflösung des Bergstaates zeigen die Wechsel im Lebensverlauf der Freiberger Professoren zwischen Wirtschaft und Wissenschaft das interdependente Verhältnis beider Bereiche.

Darüber hinaus ist im vorliegenden Artikel die Besetzung von Professuren in der Geschichte der TU Bergakademie Freiberg durch zuvor an ihr wissenschaftlich Qualifizierte unter dem Begriff Selbstrekrutierung dargelegt. Hierfür wird für die ersten einhundert Jahre der einfache Qualifikationsabschluss an der Bergakademie Freiberg zugrunde gelegt und für die Zeit nach Einführung des Promotionsrechts für die Bergakademie Freiberg die Promotion beziehungsweise Habilitation als ausschlaggebendes Moment genutzt.

Außerdem soll die regionale Herkunft auf der Grundlage der Geburtsorte für die neubestallten Professoren beziehungsweise Lehrer ausgewertet und damit Hinweisen auf die Reichweite der Bergakademie und den freien wissenschaftlichen Austausch einer für die Wissenschaftler offenen beziehungsweise eingeschränkten Gesellschaft nachgegangen werden.

Schließlich ist die zahlenmäßige Entwicklung der Professorenschaft in der Geschichte der TU Bergakademie Freiberg auf die Moderne hin abzulesen und in Bezug $\mathrm{zu}$ den bereits erforschten Studierendenzahlen zu setzen. 
Angeregt durch Fortschritte und Rückschläge bei der Exploration von Rohstoffen beziehungsweise durch die Nachfragesituation im Zuge der Industrialisierung formte sich die Bergakademie in Freiberg. In ihrer Geschichte wird der Einfluss der jeweiligen politischen Ordnung auf die sächsische Hochschule ebenso sichtbar wie kulturelle Codes des Aufstiegs ihrer Professorenschaft.

\section{Die Bergakademie Freiberg als Teil des sächsischen Bergstaates}

Vor rund 250 Jahren wurde im Frühjahr 1766 der Lehrbetrieb an der Bergakademie Freiberg aufgenommen. ${ }^{1}$ Dies geschah in personeller Kontinuität zur Stipendienkasse, über die seit 1702 montanwissenschaftliche Lehraufträge im sächsischen Bergstaat organisiert worden waren. ${ }^{2}$ Die Selbstrekrutierung des Lehrpersonals war für die Stipen-

1 Die wesentliche Grundlage der statistischen Daten stammt aus den Biografien in HARTmut Schleiff/Roland Volkmer/Herbert E. Kaden (Hg.), Catalogus Professorum Fribergensis. Professoren und Lehrer der TU Bergakademie Freiberg 1765 bis 2015, Freiberg 2015. Hier sind ,jene Professoren, die vor weniger als zehn Jahren verstorben sind“, das Publikationsjahr gilt als Zäsur, nicht aufgeführt, sodass in diesen Fällen das Personeninventar des Universitätsarchivs der TU Bergakademie als Datengrundlage genutzt wurde, ebenso bei lebenden Professoren, die ihr Einverständnis zur Publikation ihrer biografischen Angaben nicht erteilten; vgl. Hartmut Schleiff, Einführung, in: ebd., S. 10-12, hier S. 12. Zur chronologischen Ordnung der Professuren für die hier vorgelegte Auswertung vgl. ebd., S. 10 f. Die Quellengrundlage für die statistischen Daten ist so transparent. Für die Zeit bis zur Aufhebung des sächsischen Bergstaates (1869) sind auch Lehrer miteinbezogen worden, wenn ihre Positionen späteren Professuren entsprachen; vgl. ebd., S. 11. Die Abb. 2 bildet denn auch bis 1869 Lehrer und Professoren ohne Unterschied ab. Nach 1869 werden Lehrer nicht mehr miteinbezogen. Es wird nicht immer wieder erneut darauf hingewiesen, dass - wenn möglich - Medianwerte für die Berechnungen zugrunde gelegt wurden. Werte, die im vorliegenden Artikel nicht extra mit Fußnoten für Quellen und Literatur versehen sind, sind durch vorgenannte Quellen belegt. Grundlegend zum Institutionenbegriff vgl. GERHard GöHler/Rudolf Speth, Symbolische Macht. Zur institutionentheoretischen Bedeutung von Pierre Bourdieu, in: Reinhard Blänkner/Bernhard Jussen (Hg.), Institutionen und Ereignis. Über historische Praktiken und Vorstellungen gesellschaftlichen Ordnens (Veröffentlichungen des Max-Planck-Instituts für Geschichte 138), Göttingen 1998, S. 17-48, hier S. 18.

2 Im Reskript vom 26. August 1702 „zur Errichtung der Stipendienkasse beim Oberbergamt in Freiberg“ steht für die Montanwissenschaften „Bergk- und Schmelzwissenschaft“; vgl. Hans Baumgärtel/Eberhard Wächtler, Die Stipendienkasse 1702 bis 1765, in: Bergakademie Freiberg. Festschrift zu ihrer Zweihundertjahrfeier am 13. November 1965, Bd. 1: Geschichte der Bergakademie, Leipzig 1965, S. 60-62, hier S. 60. ,Montanwissenschaften' steht hier also als analytischer Begriff für einen Fächerkanon, der 1702 für die Ausbildung künftiger Bediensteter des sächsischen Bergstaates in seinem Kernbestand bereits angelegt war und mit Gründung der Bergakademie kameralistisch-naturwissenschaftlich weiter ausdifferenziert wird. Vgl. hierzu außer ebd. auch Rainer Sennewald, Die Stipendiatenausbildung von 1702 bis zur Gründung der Bergakademie Freiberg 1765/66, in: Technische Universität Bergakademie Freiberg. Festgabe zum 300. Jahrestag der Gründung der Stipendienkasse für die akademische Ausbildung im Berg- und Hüttenfach zu Freiberg in Sachsen, Freiberg 2002, S. 407-429, hier S. 410-412 und 416-419; Hans BaumgärTEL, Zur Entstehung der Bergbauwissenschaften (Von 1500-1770), Diss. Univ. Leipzig 1964, S. 177 f. Zum Begriff ,sächsischer Bergstaat', wie er auch hier genutzt wird, vgl. Hartmut Schleiff, Aufstieg und 
dienkasse schon seit den 1740 er-Jahren gegeben. ${ }^{3}$ Sie setzte sich über die Gründung der Bergakademie im Jahr 1765 hinweg fort. ${ }^{4}$ Liest man den Umfang der Selbstrekrutierung ab 1770 für fünf Generationen Professoren und Lehrer der Bergakademie ab, ${ }^{5}$ dann liegt dieser zunächst bei 60 Prozent, um fortan noch höher und ab 1830 für die nächsten drei Generationen gar bei über 90 Prozent zu liegen. ${ }^{6}$ Nicht nur die Professoren und Lehrer der ersten Stunde waren zuvor Stipendiaten beziehungsweise zum Teil Lehrer der Stipendienkasse gewesen, ${ }^{7}$ sondern auch gut ein Viertel der ersten Bergakademisten war zuvor über die Stipendienkasse gefördert unterrichtet worden. ${ }^{8}$

Während in den rund dreieinhalb Generationen bis zur Aufnahme des Lehrbetriebs an der Bergakademie durchschnittlich nicht ganz zwei Stipendiaten pro Jahr ihren Abschluss gemacht hatten, ${ }^{9}$ wurden nach der Gründung der Bergakademie nur wenig mehr künftige Bedienstete des sächsischen Bergstaates ausgebildet. Bis zu dessen Aufhebung, das heißt für die ersten rund fünf Generationen Studenten an der Bergakademie, blieb die Zahl derartiger Neuimmatrikulationen je Jahr zumeist unter

Ausbildung im sächsischen Bergstaat zwischen 1765 und 1868, in: Ders./Peter Konečný (Hg.), Staat, Bergbau und Bergakademie. Montanexperten im 18. und frühen 19. Jahrhundert (Vierteljahrschrift für Sozial- und Wirtschaftsgeschichte, Beihefte 223), Stuttgart 2013, S. 125-159, hier S. 126.

3 Vgl. Sennewald, Stipendiatenausbildung (wie Anm. 2), S. 425.

4 Vgl. für den Übergang zur Bergakademie und die ersten hundert Jahre nach ihrer Gründung: Schleiff, Aufstieg und Ausbildung (wie Anm. 2), S. 141, Abb. 2.

5 Eine Generation ist hier auf 20 Jahre festgelegt.

6 Der bei Schleiff, Aufstieg und Ausbildung (wie Anm. 2), S. 142, aus der Auswertung herausragender Einzelwissenschaftler abgeleitete Aussage, „dass hier die Entwicklung hin zur Auswahl des Lehrpersonals in offener Konkurrenz" vollzogen wurde, muss das aus der statistisch gemittelten Professorenschaft erlangte Ergebnis für die hohe Selbstrekrutierung kritisch beiseite gestellt und für den Durchschnitt der Lehrer und Professoren der ersten hundert Jahre zurückgewiesen werden; dort mit Bezug auf PETER Moraw, Gesammelte Beiträge zur Deutschen und Europäischen Universitätsgeschichte. Strukturen - Personen - Entwicklungen, Leiden/Boston 2008, S. 52. Der daraus abzuleitenden Signifikanz für die frühneuzeitliche Universität in Deutschland - vgl. Marita Baumgarten, Professoren und Universitäten im 19. Jahrhundert. Zur Sozialgeschichte deutscher Geistes- und Naturwissenschaftler (Kritische Studien zur Geschichtswissenschaft 121), Göttingen 1997, S. 17 - steht der vor allem zur Zeit des Deutschen Kaiserreichs und abgemildert auch noch zur DDR-Zeit hohe Anteil an Sachsen unter den Professoren an der Bergakademie Freiberg entgegen; vgl. weiter unten.

7 Vgl. hierzu Baumgärtel/Wächtler, Stipendienkasse (wie Anm. 2), S. 61, für Johann Friedrich Wilhelm Charpentier, Christlieb Ehregott Gellert, Christoph Hieronymus Lommer, Johann Andreas Klotzsch und Christlieb Ehregott Gellert (dieser war zuvor Lehrbeauftragter der Stipendienkasse wie auch der zuvor genannte Klotzsch).

8 Vgl. zum Beispiel für Johann Friedrich Freiesleben und Johann Friedrich Wilhelm Charpentier, der zugleich Professor an der Bergakademie war, ebd., S. 61. Insgesamt zu den Studenten, die im Frühjahr 1766 ihr Studium aufnahmen, vgl. Universitätsarchiv TU Bergakademie Freiberg (im Folgenden: UA Freiberg), OBA, Sign. 236, fol. 48-56v. Ein Abdruck der Namen findet sich in Carl GotTlieb GotTschalk, Verzeichniss Derer, welche seit Eröffnung der Bergakademie und bis zum Schluss des ersten Säculum's auf ihr studirt haben, in: Festschrift zum hundertjährigen Jubiläum der Königl. Sächs. Bergakademie zu Freiberg am 30. Juli 1866, Dresden 1866, S. 221-295, hier S. 223. Vergleicht man diese mit BAumgärTEL, Entstehung der Bergbauwissenschaften (wie Anm. 2), S. 241-244, ergibt sich obiger Wert.

9 Vgl. BAumgäRTel, Entstehung der Bergbauwissenschaften (wie Anm. 2), S. 241-244. 


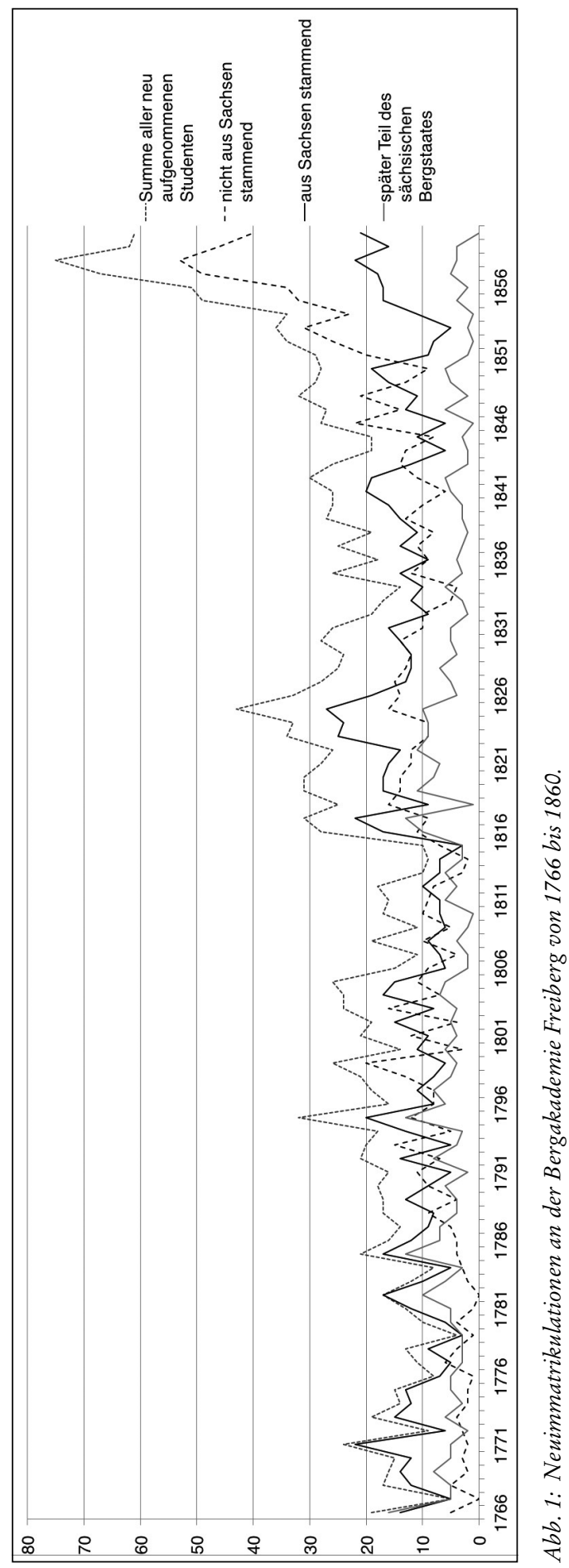


zehn. ${ }^{10}$ Obgleich sich die Zahl dieser Stipendiaten an der Bergakademie, das heißt jener, die - wie schon bei der Stipendienkasse - ihre Förderung mit ihrem Dienst im sächsischen Bergstaat abzugelten hatten, ${ }^{11}$ relativ vervielfachte, blieb ihre absolute Zahl gering.

Hatten die Lehrer, die über die Stipendienkasse finanziert worden waren, zwar auch schon Studenten aus dem deutschen und aus dem nichtdeutschen Ausland unterrichtet, so institutionalisierte sich die montanwissenschaftliche Ausbildung auch für ausländische Studenten mit Gründung der Bergakademie weiter. Dem Wissenstransfer zwischen den unterschiedlichen Bergbaurevieren Deutschlands, aber auch Europas, Nord- und Südamerikas sowie später Afrikas und dann auch Asiens stand somit langfristig Tür und Tor offen. ${ }^{12} \mathrm{Ab}$ dem zweiten Drittel des 19. Jahrhunderts, also nach knapp dreieinhalb Generationen, war die Zahl jener Neuimmatrikulierten, die künftig nicht ihren Dienst im sächsischen Bergstaat versehen sollten, um deutlich mehr als den Faktor 2 höher. Ausländer und andere frei immatrikulierte Studenten überwogen nun tendenziell um ein höheres Vielfaches (Abb. 1). ${ }^{13}$

Werden beide Studentengruppen zusammengerechnet - 1826 waren dies insgesamt 82 Studenten ${ }^{14}$ - und auf die Professoren beziehungsweise Lehrer bezogen, ergibt dies für die ersten vier Generationen ein Verhältnis per anno von rund einem Professor beziehungsweise Lehrer auf vier neuimmatrikulierte Studenten. Erst mit der fünften Generation änderte sich dieses Verhältnis nachhaltig auf eine größere Spreizung, das heißt hier zunächst auf ein Verhältnis von 1:8. Ab dieser Zeit pendelte sich der Lehrkörper auf durchgängig mehr als zehn Professoren beziehungsweise Lehrer ein.

\section{Die Bergakademie Freiberg von der Aufhebung des sächsischen Bergstaates bis zur Weimarer Republik}

Mit der Aufhebung des Bergstaates und damit dem Wegfall des Unterstellungsverhältnisses der Bergakademie zum Oberbergamt im Jahr 1869 verlor die Bergakademie ihre Funktion als kameralistisch-naturwissenschaftliche Lehr- und Forschungsanstalt des Bergstaates und stellte sich nunmehr nachdrücklich auf eine bildungsbürgerliche Lehrund Forschungseinrichtung mit freier Immatrikulation um. ${ }^{15}$ Dieser Prozess begann

10 Vgl. Schleiff, Aufstieg und Ausbildung (wie Anm. 2), S. 148.

11 Vgl. UA Freiberg, OBA 1 (Altsignatur: 65), fol. 1v. Die Stipendienkasse ist Mitte März 1766 in der finanziellen Ausstattung der Bergakademie aufgegangen; vgl. HANs BAUMGÄRTEL, Die Gründung der Bergakademie, in: Bergakademie Freiberg (wie Anm. 2), Bd. 1, S. 73-80, hier S. 78.

12 Vgl. für die absoluten Zahlen der Neuimmatrikulierten und für das Verhältnis deutscher zu nichtdeutschen Ausländern GotTschalk, Verzeichnis (wie Anm. 8), S. 293-295. Zu den Schwierigkeiten, die absoluten Zahlen aller Studierenden, also nicht nur der Neuimmatrikulierten, pro Jahr zu ermitteln, vgl. Hans BAumgärTEL, Aus der Geschichte der Bergakademie Freiberg, Berlin ${ }^{3} 1961$, S. 76, wie auch OTfRIED WAgENBRETH u. a., Die Technische Universität Bergakademie Freiberg und ihre Geschichte, Freiberg 32012, S. 53.

13 Vgl. Schleiff, Aufstieg und Ausbildung (wie Anm. 2), S. 148. Hier ist der Anteil jener, die später im sächsischen Bergstaat dienten, für das Jahr ihrer Immatrikulation an der Summe aller Neuimmatrikulationen der Bergakademie erstmals ausgewiesen. Zu dieser Studentengruppe vgl. ebd., S. 156.

14 Vgl. Eberhard Wä́chtler/Fritz Zillmann, Die Freiberger Studentenschaft 1765 bis 1945, in: Bergakademie Freiberg (wie Anm. 2), Bd. 1, S. 274-288, hier S. 282.

15 Vgl. Schleiff, Aufstieg und Ausbildung (wie Anm. 2), S. 158. 
bereits mit dem zweiten Drittel des 19. Jahrhunderts. ${ }^{16}$ Im Zuge der Zeunerschen Reformen wurde die Bergakademie um 1871 wissenschaftsorganisatorisch neu ausgerichtet. Neben neuen Kostenordnungen für ein Studium in Freiberg - zu dieser Zeit fiel auch die besonders geförderte Gruppe Studierender weg, die sich zu einer künftigen Arbeit im Bergstaat verpflichtet hatten - wurde der Schwerpunkt der Lehre und Forschung auf neue Rohstoffe, aber auch auf neue Fächer gelegt. ${ }^{17}$ Die Personalunion von Lehrenden und Offizianten mit weiteren Funktionen im Bergstaat, die zwischen 1766 und 1868 im Durchschnitt Dreiviertel betrug, ${ }^{18}$ entfiel 1869. Auch sank der prozentuale Anteil unter den neu eingestellten Professoren beziehungsweise Lehrern, die in Sachsen geboren waren. Lag dieser für die ersten rund einhundert Jahre Lehrbetrieb an der Bergakademie (1766-1871) insgesamt bei knapp vier Fünfteln, betrug er für die Zeit des Deutschen Kaiserreichs nur noch drei Fünftel.

Die Ausbildung war in Freiberg zu dieser Zeit nicht so einseitig, wie es in Preußen neun Jahre nach Gründung der Berliner Bergakademie tönte. Anders als in der Berliner Börsenzeitung vom 7. September 1869 dargestellt, war die Bergakademie Freiberg nicht nur eine Akademie für den Silber- und Bleibergbau. ${ }^{19}$ Vielmehr standen zum Beispiel die Freiberger Professoren Karl Amandus Kühn und Friedrich August Breithaupt für die Förderung der Steinkohle in Sachsen. Sie waren bereits Ende der 1830erJahre an der Gründung eines der größten Steinkohlen-Aktienvereine im Zwickauer Revier beteiligt gewesen und hatten im Jahr 1842 für den Nachweis der Steinkohlenführung jenseits der Oberhohndorfer Verwerfung bei Zwickau die Große Goldene

16 Dies lässt sich auch daran ablesen, dass erst ab dieser Zeit an der Bergakademie anhaltend eine deutlich mehr als doppelt so hohe Neuimmatrikulation von Studenten erfolgte, die nicht mehr dafür vorgesehen waren, im sächsischen Bergstaat zu dienen; vgl. ebd, S. 148. Vgl. auch SchleIfF, Einführung, in: Schleiff/Volkmer/Kaden, Catalogus Professorum Fribergensis (wie Anm. 1), S. 11.

17 Vgl. Hans BaumgäRtel, Gustav Zeuner und die Reorganisation der Bergakademie 1872-1875, in: Bergakademie. Zeitschrift für Bergbau, Hüttenwesen und verwandte Wissenschaften 10 (1957), S. 534-537, hier S. 536. Ebd. wird auf neue Fächer wie metallurgisch-mechanische Technologie, chemische Technologie, Feuerungskunde verwiesen, für die Neuetablierung der Eisenhüttenkunde in dieser Zeit vgl. STEFAN Krebs, Genese und Struktur eines technikwissenschaftlichen Feldes. Über den Kampf der Aachener Eisenhüttenkunde um Macht und Autonomie 1870-1914, Diss. Technische Hochschule Aachen 2008, S. 36, sowie Bernd Lychatz/Ralf-Peter Bösler (Hg.), Die Freiberger Eisenhüttenkunde. Ein historischer Abriss mit biografischen Skizzen, Freiberg 2014.

18 Vgl. Chursächsischer Bergwerks-Calender 1773, 1774, 1778-1780; Bergmännischer Kalender 1790, 1791; Freybergischer Stadt-, Land und Berg-Kalender 1795-1868; Kalender für den Sächsischen Berg- und Hüttenmann 1827-1829; Jahrbuch für den Berg- und Hüttenmann 1830-1868; Die Bergakademie zu Freiberg. Zur Erinnerung an die Feier des hundertjährigen Geburtstages Werner's am 25. September 1850, Freiberg o. J.; GotTschalk, Verzeichnis (wie Anm. 8).

19 Berliner Börsenzeitung vom 7. September 1869 nach BAumgäRTEL, Gustav Zeuner (wie Anm. 17), S. 535. Wenn Baumgärtel hier zu dem Schluss kommt, dass der Artikel „zwar nicht unparteiisch, aber objektiv richtig“ sei, dann steht dies im Gegensatz zu obigen Aussagen. Anders als in Berlin war das Wissen, dass im sächsischen Bergstaat mehr als nur die oben genannten Rohstoffe - u. a. auch Kohle - abgebaut wurden, in London so populär, dass es sogar im Reiseführer stand; vgl. John Murray, A hand-book for travellers on the continent. Being a guide through Holland, Belgium, Prussia and Northern Germany, and along the Rhine, from Holland to Switzerland, London 21838, S. 420. 
Gewerbsverdienst-Medaille Sachsens erhalten. ${ }^{20}$ Für diese Exploration konnten sie auf den Arbeiten der Bergakademie zur Landesuntersuchung aufbauen, die seit der ersten Generation Lehrer und Professoren an der Bergakademie die Rohstoffe Sachsens kartierte - und dies seit 1788 mit einem besonderen Augenmerk auf Steinkohlevorkommen. ${ }^{21}$ Auch Kühns Nachfolger in der Lehre der Geognosie, Prof. Dr. Carl Friedrich Naumann, setzte die Landesuntersuchung insbesondere mit Bezug auf die Kohlevorkommen Sachsens fort. ${ }^{22}$ Es ist sicher auch seiner diesbezüglichen Arbeit zuzuschreiben, dass er dann 1842 auf eine neu geschaffene Professur in Leipzig berufen wurde. Hieran wird jedoch auch deutlich, dass der Bergakademie Freiberg akademische Konkurrenz auf ihrem ureigensten wissenschaftlichen Terrain erwuchs. Nicht unerwähnt bleiben können die Verdienste des Freiberger Professors für Physik, Ferdinand Reich, und des Freiberger Professors für Lötrohrprobierkunde, Hieronymus Theodor Richter, bei der Entdeckung des chemischen Elements Indium im Jahr $1863 .{ }^{23}$ Sie ist ein Ausweis des Entwicklungsstandes der naturwissenschaftlichen Forschung. Diese war an der Bergakademie in den vorangegangenen Jahren massiv personell gefördert worden und fand in der Entdeckung des Germaniums 1886 an der Bergakademie Freiberg eine Fortsetzung. ${ }^{24}$

Reformdruck auf die Bergakademie war rund fünfzehn Jahre zuvor nicht zuletzt an den einbrechenden Studentenzahlen deutlich geworden, ${ }^{25}$ studierten doch im Jahrgang

20 Vgl. Gisela-Ruth Engewald, Friedrich August Breithaupt (1791-1873). Mineraloge und Unternehmer, in: Hans Prescher (Hg.), Leben und Wirken Deutscher Geologen im 18. und 19. Jahrhundert, Leipzig 1985, S. 210-246, hier S. 227-232; die Gründung des Aktienvereins wurde zum 3. Februar 1840 genehmigt.

21 Vgl. Hartmut Schleiff, Knowledge Practices in the Establishment and Reproduction of the Mining Elite in Saxony, 1765-1868, in: André Holenstein/Hubert Steinke/Martin Stuber (Hg.), Scholars in Action. The Practice of Knowledge and the Figure of the Savant in the 18th Century, Bd. 1, Leiden/Boston 2013, S. 827-851, hier S. 841; OTFRIED Wagenbreth, Der sächsische Mineraloge und Geologe Carl Friedrich Naumann (1797-1873), in: Abhandlungen des Staatlichen Museums für Mineralogie und Geologie zu Dresden 29 (1979), S. 313-396, hier S. 335.

22 Vgl. ebd., S. 315, 335 f., 339 und 358-362.

23 Vgl. Mike Haustein/Herbert A. Schneider/Klaus Volke, Über die in Freiberg entdeckten chemischen Elemente, in: Dietrich Stoyan (Hg.), Bergakademische Geschichten. Aus der Historie der Bergakademie Freiberg erzählt anlässlich des 250. Jahrestages ihrer Gründung, Freiberg 2015, S. 135-156, hier S. 140-145.

24 Vgl. ebd. auch zur personellen Kontinuität von Clemens Winkler, S. 135 und 141. Es waren jedoch nicht nur diese Fächer, die konkurrenzfähig aufgestellt waren, sondern auch die Geologie, Lagerstättenlehre, Metallhüttenkunde, Markscheidekunde und Geophysik; vgl. hierzu auch HeINz BëssLER u. a., Grundzüge der gesellschaftlichen Entwicklung und des wissenschaftlich-technischen Fortschritts, in: Bergakademie Freiberg (wie Anm. 2), Bd. 1, S. 167-174, hier S. 169. Diese und obige Argumentation stehen in Widerspruch zu Eberhard Wächtler, Der Niedergang der Bergakademie in den 50er und 60er Jahren des 19. Jahrhunderts und seine Ursachen, in: ebd., S. 160-163, hier S. $160 \mathrm{f}$.

25 Baumgärtel, Gustav Zeuner (wie Anm. 17), S. 536, behauptete, dass die Krise der Bergakademie, die mit den Zeunerschen Reformen aufgehoben worden sei, an der Verlagerung der Studierendenzahlen auf Ausländer abgelesen werden könne, da diese zwar Geld, aber keine Vorkenntnisse mitgebracht hätten. Dies steht im Widerspruch zu ihrem weiterhin hohen Anteil in den Jahren nach den Zeunerschen Reformen. Vgl. hierzu Wächtler/Zillmann, Freiberger Studentenschaft (wie Anm. 14), S. 285: „Seit dem Studienjahr 1895/96 studierten an der Bergakademie bis zum Ersten Weltkrieg stets mehr Ausländer als Deutsche.“ 
1870/71 rund 70 Prozent weniger als im Jahrgang 1861/62. ${ }^{26}$ Hierfür war die neu entstandene Konkurrenz einer höheren naturwissenschaftlich-technischen Bildung, zum Beispiel in Clausthal, Berlin, ${ }^{27}$ Aachen, Karlsruhe, Zürich und Dresden, ebenso verantwortlich ${ }^{28}$ wie die Verlagerung der industriellen Entwicklung auf neu entstehende Wirtschaftszweige, die eines neuen naturwissenschaftlich-technischen Wissens bedurften. ${ }^{29}$ Der Standortvorteil der Bergakademie Freiberg im politisch-wirtschaftlichen Zentrum des Bergstaates entfie ${ }^{30}$ auch für die Montanwissenschaften, verlor der Silberbergbau im Freiberger Revier doch noch im Laufe des Kaiserreichs an Relevanz. ${ }^{31}$ Auch die größeren Kohlevorkommen Sachsens lagen jenseits der Grenzen des Erzgebirges. Naturwissenschaftlich-technische Forschung und Lehre konnte sich zum Beispiel für das Eisenhüttenwesen, für den Maschinenbau, die optische und chemische Industrie ${ }^{32}$ in den verkehrstechnisch günstiger erschlossenen Städten, die politische, kulturelle, wirtschaftliche und wissenschaftliche Zentren waren beziehungsweise wurden, besser etablieren.

26 Vgl. Baumgärtel, Gustav Zeuner (wie Anm. 17), S. 534. Frauen nahmen erst im Laufe des Kaiserreichs ein Studium an der Bergakademie auf; vgl. Birgit Seidel, „... diese Smith!“, in: Zeitschrift der Freunde und Förderer der Technischen Universität Bergakademie Freiberg 17 (2010), S. 181 f.

27 Durch „die Eröffnung der Bergakademie Berlin (1860) und die Erhebung der Schule in Clausthal zur Bergakademie (1864) [sind] Konkurrenzunternehmen entstanden [...], die ihre Studenten nach neuzeitlicheren Gesichtspunkten und billiger ausbilden als Freiberg"; BAUMgäRTEL, Gustav Zeuner (wie Anm. 17), S. 536.

28 Diese neu entstandene akademische Konkurrenz in Deutschland würde auch erklären, warum die Zahlen der deutschen Studenten an der Bergakademie Freiberg einbrechen. Vgl. zum Beispiel für Dresden Reiner Pommerin, 175 Jahre TU Dresden, Bd. 1: Geschichte der TU Dresden 1828-2003, Köln u. a. 2003, S. 48 f. Hinzu kommt die vereinfachte Freizügigkeit und Zulassung im Zuge der Reichseinigung. Vgl. für die steigenden absoluten Zahlen von $1864 \mathrm{zu} 1873 \mathrm{im}$ Spektrum der auch an der Bergakademie Freiberg gelehrten Fächer an den Universitäten und technikwissenschaftlichen Instituten der deutschen Länder beziehungsweise des Deutschen Kaiserreichs FrITZ RINGER, A Sociography of German Academics, 1863-1938, in: Central European History 25 (1992), S. 251-280, hier S. 254-256.

29 Vgl. Wächtler, Niedergang der Bergakademie (wie Anm. 24), S. 162. Mit dem vierten Quartal 2016 liegt aktuell und damit rechtzeitig vor Drucklegung dieses Aufsatzes vor: Helmuth Albrecht, Die Bergakademie Freiberg. Eine Hochschulgeschichte im Spiegel ihrer Jubiläen 1765 bis 2015, Halle 2016. Dort wird herausgearbeitet, dass im Jahrzehnt nach 1875 anders als an den deutschen Technischen Hochschulen insgesamt, die „,vorübergehend über die Hälfte ihrer Studierenden verloren, [...] die Bergakademie Freiberg ihre Hörerzahl kontinuierlich zu steigern" vermochte. Ursächlich ist hierfür die Wirtschaftskrise nach dem Börsenkrach des Jahres 1873 benannt; vgl. ebd., S. 159.

30 Vgl. Adolf Soetbeer, Edelmetall-Produktion und Werthverhältniss zwischen Gold und Silber seit der Entdeckung Amerika's bis zur Gegenwart, Gotha 1879, S. 21 und 33; Schleiff, Aufstieg und Ausbildung (wie Anm. 2), S. $144 \mathrm{f}$.

31 Vgl. Erwin Papperitz, Geschichte, gegenwärtige Organisation und Statistik der Bergakademie Freiberg, in: Die Königlich Sächsische Bergakademie zu Freiberg und die Königliche geologische Landesanstalt nebst Mitteilungen über die Entwickelung und den Stand des Berg- und Hüttenwesens und der Bergpolizei im Königreiche Sachsen, Freiberg 1904, S. 1-26, hier S. 1. Einige Jahre nach Erscheinen der Publikation kam der Erzbergbau im Erzgebirge zwischenzeitlich zum Erliegen; vgl. ERwin PAPPeritz, Gedenkschrift zum Hundertfünfzigjährigen Jubiläum der Königlich Sächsischen Bergakademie zu Freiberg, Freiberg 1916.

32 Vgl. Wächtler, Niedergang der Bergakademie (wie Anm. 24), S. 162. 
Dennoch gelang es - nicht zuletzt durch die Zeunerschen Reformen - das akademische Know-how für die Natur- und Technikwissenschaften in Freiberg zu erhalten und sogar leicht auszubauen. Und das nicht nur für diese Fachbereiche, sondern es kam in der ersten Generation im Deutschen Kaiserreich in Bezug zu der vorangegangenen auch zu einer Verdoppelung des Anteils der Juristen und Wirtschaftswissenschaftler an der Gesamtprofessorenschaft. Bis zur Aufhebung des Bergstaates betrug ihr Anteil - einschließlich der für die Unterrichtung im Verwaltungshandeln Tätigen - noch knapp 9 Prozent. ${ }^{33}$ Der Anteil Professoren für die Naturwissenschaften und Mathematik war zwischen 1827 und 1864 angestiegen und lag deutlich über dem Anteil der Ingenieurwissenschaftler. Bis 1871 stieg dann der Anteil für die technischen Wissenschaften stark. Bald darauf - also nach den Zeunerschen Reformen, Gustav Zeuner selbst wechselte schon Mitte der 1870er-Jahre nach Dresden zur neuerstarkenden Konkurrenz, dem Polytechnikum - glichen sich beide Bereiche an.

Mit Zeuners Berufung in Freiberg und den Reformen war es auch zu einem starken Anstieg an Promovierten bei den neu berufenen Professoren gekommen. Ihr Anteil im Jahresmittel an allen Professoren lag während des Deutschen Kaiserreichs bei knapp 60 Prozent und stieg damit im Vergleich zur letzten Generation im Bergstaat um knapp 30 Prozent. Im Bergstaat hatte ihr gemittelter Anteil bis zum ersten Drittel des 19. Jahrhunderts bei niedrigen absoluten Zahlen sogar nur bei unter 14 Prozent pro Jahr gelegen.

Im Zuge der Zeunerschen Reformen war die Diplomprüfung eingeführt worden, die ab 1901 für die Aspiranten des höheren technischen Staatsdienstes in der Berg-und Hüttenverwaltung als erste Staatsprüfung galt und um eine Assessorprüfung, also eine zweite Staatsprüfung, ergänzt wurde. Seit 1901 war das Habilitationsverfahren an der Bergakademie geregelt. ${ }^{34}$ Mit dem 27. Februar 1903 sind die Diplomingenieurprüfungen an der Bergakademie denen an den Technischen Hochschulen gleichgestellt worden. Zwei Jahre darauf konnten Promotionsprüfungen in Freiberg abgelegt werden. So wurde es auch auf den Promotionsurkunden festgehalten, der Rechtsakt selbst erfolgte jedoch noch durch die Technische Hochschule Dresden. Erst ab 1920 konnten dann an der Bergakademie Promotionsverfahren vollständig abgeschlossen werden. ${ }^{35}$ Es wurde nun auch ohne die Technische Hochschule Dresden der akademische Titel ,Dr.Ing. verliehen. Das Promotionsrecht erlangte die Bergakademie also in Stufen leicht verzögert. ${ }^{36}$ Der eigenständigen Entwicklung der Naturwissenschaften an der Bergakademie kam man mit dem Recht der Vergabe des akademischen Titels ,Dr. rer. nat.“ erst 1939 nach. ${ }^{37}$ In der NS-Zeit ist die Habilitation für das Deutsche Reich einheitlich geregelt und „mit Dr. habil. eine Trennung zwischen Lehrbefähigung und Lehrbefugnis, also zwischen Eignung und Bedarf“ eingeführt worden. ${ }^{38}$

Zur bergakademischen Unterrichtung im Verwaltungshandeln vgl. ScHLEIFF, Aufstieg und Ausbildung (wie Anm. 2), S. 133, 144 und 151-155.

34 Vgl. UA Freiberg, D/R, Sign. 12; und Sammlungen/Urkunden etc., Sign. Y 8.

35 Vgl. Baumgärtel, Geschichte der Bergakademie (wie Anm. 12), S. 49.

36 Zur Entwicklung des Promotionsrechts der Technischen Hochschulen vgl. Helmuth Albrecht, Technische Bildung zwischen Wissenschaft und Praxis. Die Technische Hochschule Braunschweig 1862-1914, Hildesheim 1987, S. 347-349.

37 Vgl. zu den Jahren der Einführung akademischer Abschlüsse an der Bergakademie Hans Baumgärtel, Der Weg zur modernen Hochschule, in: Bergakademie Freiberg (wie Anm. 2), Bd. 1, S. 175-178, hier S. 176 und 178.

38 RüDIgER vOM BRUCH, Qualifikation und Spezialisierung. Zur Geschichte der Habilitation, in: Forschung \& Lehre 2000, H. 2, S. 69 f., hier S. 70, mit Bezug auf die Reichshabilitationsordnung von 1939. Vgl. für eine frühere Datierung dieser Regelung auch Franz BACHÉr, Die Reichshabilitationsordnung, in: Deutsche Wissenschaft, Erziehung 
Die staatliche Vereinheitlichung der akademischen Abschlüsse blieb nicht ohne Auswirkungen auf die Zahl der Studierenden an der Bergakademie. Schon die Einführung der Staatsprüfung im Jahr 1860 stand im Zusammenhang mit gestiegenen Studentenzahlen. Ein Jahr nach ihrer Einführung studierten rund 150 Studenten an der Bergakademie, das heißt rund ein Drittel mehr als in den 1850er-Jahren. ${ }^{39}$ Zwischen 1899 , dem Jahr der Einführung des Wahlrektorats, und 1914, dem Beginn des Ersten Weltkriegs, kam es im Verhältnis zu den zehn Jahren vor 1899 zu mehr als einer Verdoppelung der Studierendenzahl. Der tiefe Einschnitt der Zahl um rund die Hälfte im Jahresmittel während des Ersten Weltkriegs konnte schon zu Beginn der Weimarer Republik geheilt werden: 1919 fehlten nur wenig mehr als 10 Studierende, es waren jetzt 419, um auf den Wert des Jahres vor Beginn des Ersten Weltkriegs zu kommen.

\section{Professoren und Studierende an der Bergakademie Freiberg in Weimarer Republik, NS-Diktatur, DDR und Bundesrepublik Deutschland}

In der Weimarer Republik stieg die Studierendenzahl trotz politischer Krisen zwischen 1919 und 1923 auf im Jahr 1922 hohe 685 und im Jahr 1923 gar auf 692. Danach fielen die Zahlen in großen Schritten bis zur Weltwirtschaftskrise von 1929 auf 245, um im ersten Jahr der NS-Diktatur bei nur noch $173 \mathrm{zu}$ liegen. Die Professuren nahmen hingegen leicht zu. Ihre Zahl lag ab 1928 bis zum Ende der Weimarer Republik um Mitte Zwanzig, sodass sich zu dieser Zeit das Verhältnis Professor pro Student stetig verbesserte. Diese Tendenz setzte sich am Anfang der NS-Diktatur weiter fort. Die Studierendenzahlen stiegen erst wieder mit dem Beginn des Zweiten Weltkriegs beziehungsweise nach einem kurzen Einbruch im Jahr 1940 dann wieder mit dem Überfall des Deutschen Reichs auf die Sowjetunion. Von diesem Jahr, also von 1941, kletterten sie bis 1945 auf 282. Lag mit Beginn der Weimarer Republik die Zahl der lehrenden Professoren noch knapp unter 20, fiel sie nach 1923 bis zum Ende des Zweiten Weltkriegs nicht mehr unter 20 (Abb. 2). Die durchschnittliche Anzahl lehrender Professoren pro Jahr wuchs für die Weimarer Republik und die NS-Zeit zusammengenommen gegenüber ihrer Anzahl im Kaiserreich um 70 Prozent. ${ }^{40}$

und Volksbildung. Amtsblatt des Reichsministeriums für Wissenschaft, Erziehung und Volksbildung und der Unterrichtsverwaltung der Länder 1 (1935), H. 24, Nichtamtlicher Teil, S. 17 f., hier S. 17, mit Bezug auf die Reichshabilitationsordnung vom 13.12.1934 in ebd., Amtlicher Teil, S. 12-14. Dort ist im $\$ 2$ der ,akademische Grad eines habilitierten Doktors“ geregelt; vgl. so auch MichaEL Jung, „Voll Begeisterung schlagen unsere Herzen zum Führer“. Die Technische Hochschule Hannover und ihre Professoren im Nationalsozialismus, Norderstedt 2013, S. 81 f.

39 Bei Albrecht, Bergakademie Freiberg (wie Anm. 29), S. 139, wird der starke Rückgang nichtsächsischer Studenten an der Bergakademie Freiberg nach 1866, also sechs Jahre nach Einführung der Staatsprüfung, vor allem darauf zurückgeführt, dass Preußen die Freiberger Staatsprüfungen nicht anerkannte. Vgl. für die Studierendenzahlen Wächtler/ZillmanN, Freiberger Studentenschaft (wie Anm. 14).

40 Vgl. für die Studierendenzahlen Wëchtler/Zillmann, Freiberger Studentenschaft (wie Anm. 14). Erstmalig liegt hier eine quantitative Auswertung der Professorenschaft für die ersten 250 Jahre Geschichte der TU Bergakademie Freiberg vor. Es gibt jedoch Ergebnisse für einzelne Teilaspekte, wie die Geschichte einzelner Fachbereiche in bestimmten Zeitabschnitten, die nicht aneinandergereiht und aufsummiert werden können; vgl. für die Zeit von 1946 bis 1960 zum Beispiel BAumgäRTEL, Geschichte der Bergakademie (wie Anm. 12), S. 61. Die geringen Abweichungen zu oben genannten Zahlen können, da sie weitgehend mit den hier ausgewerteten Daten für den dort 


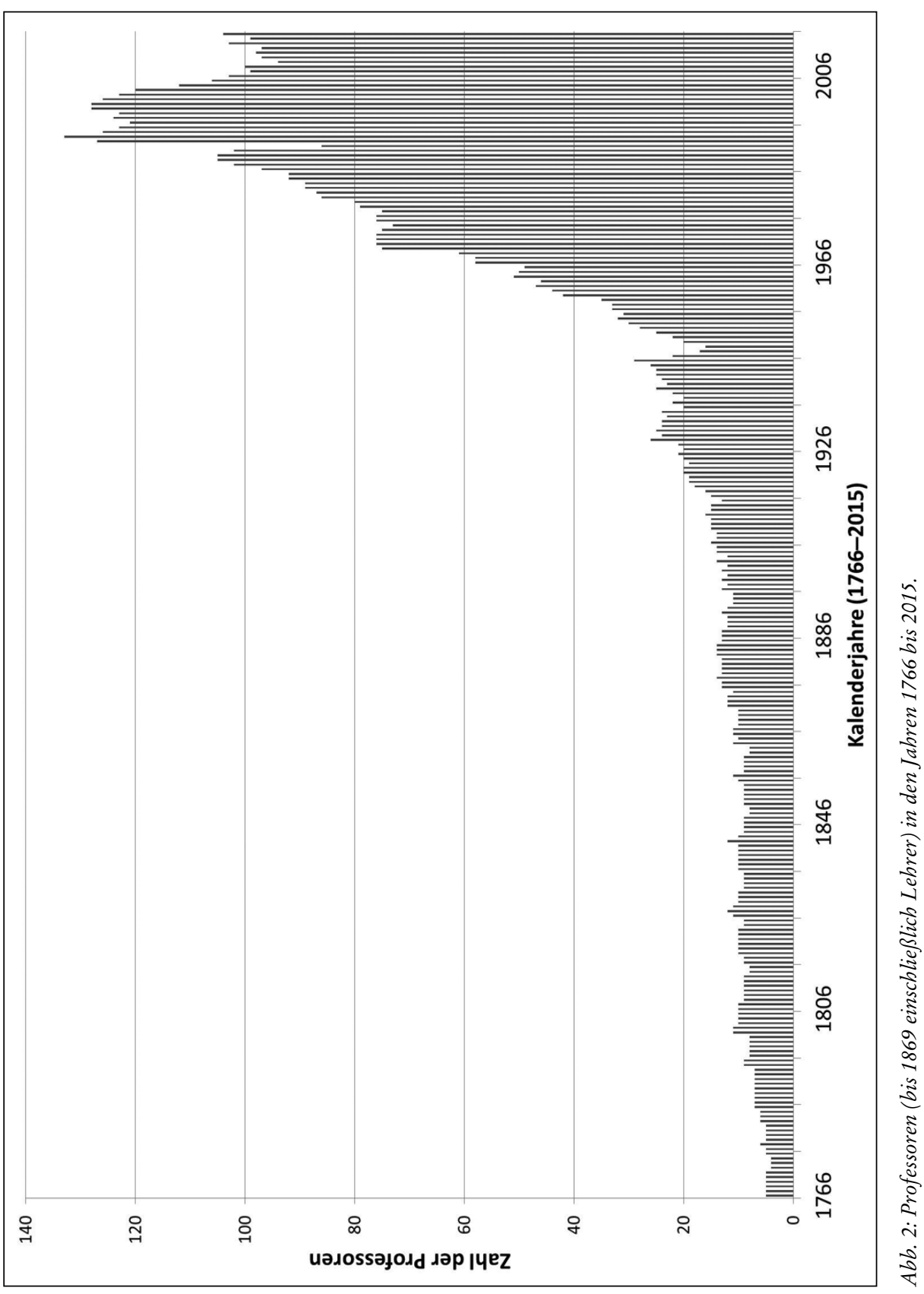


Die Standardisierung der Qualifikationsabschlüsse zeigt sich auch an der Steigerung des pro Jahr gemittelten Anteils an Professoren, die als wissenschaftliche Qualifikation eine Promotion abgelegt hatten. Betrug ihr Anteil in der Weimarer Republik noch 71,6 Prozent, stieg dieser Wert in der NS-Zeit auf 84,9 Prozent.

Die Habilitation als Qualifikation für Professoren etablierte sich an der Bergakademie erst allmählich in der letzten Generation vor Beginn des 20. Jahrhunderts. In diesen 20 Jahren lag ihr Anteil bei 7,7 Prozent. Ab dieser Zeit gab es an der Bergakademie immer Professoren, die sich habilitiert hatten. Ihr Anteil nahm nach und nach zu. Ende des ersten Jahrzehnts des 20. Jahrhunderts überstieg er endlich 10 Prozent an der Gesamtprofessorenschaft und stieg dann bis zum Beginn des Ersten Weltkriegs auf 20 Prozent. Noch vor Ende des Krieges hatte sich dieser Wert auf rund 30 Prozent eingepegelt. Vom Mittelwert für das Deutsche Kaiserreich (10) zu dem für die Weimarer Republik (41) fand eine Steigerung um rund das Vierfache statt, und im Vergleich von Weimarer Republik zur NS-Zeit (61) nahm der Anteil Habilitierter an den Professoren nochmals zu, und zwar nunmehr um die Hälfte.

Das Habilitationsalter lag für die Bergakademieprofessoren im Deutschen Kaiserreich gemittelt bei 28 , stieg danach auf rund 30 beziehungsweise für die NS-Zeit 33 Jahre ${ }^{41}$ und, um einen Ausblick zu geben, in der DDR auf gerundet 38 sowie in der Bundesrepublik auf knapp 40 Jahre. Zwischen Deutschem Kaiserreich und Bundesrepublik stieg das Habilitationsalter also um rund 43 Prozent. Vergleicht man diesen Wert mit dem Wert der Zunahme des Promotionsalters für den gleichen Zeitraum, dann hat das Habilitationsalter um mehr als doppelt soviel zugenommen wie das Promotionsalter. Das auf das Jahr gemittelte Promotionsalter der neuberufenen Professoren beträgt für das Deutsche Kaiserreich und die Weimarer Republik um die 25,5 Jahre und stieg für die NS-Zeit auf 26,2, die DDR auf 29,7 und die Bundesrepublik auf 30,3 Jahre. Hierbei ist die allgemeine Beobachtung in Rechnung zu stellen, dass die Qualifikationsschriften bis zur Wiedervereinigung zumeist im zweistelligen Seitenumfang blieben. Im Untersuchungszeitraum nimmt die Spreizung zwischen Promotionsalter und Habilitationsalter generell zu.

Die Anteile der Professoren nach Fachbereichen zeigen für die NS-Zeit einen starken Anstieg der Technikwissenschaften von 45 Prozent im Jahr 1935 auf mehr als 61 Prozent im Jahr 1944. Diese Tendenz setzte sich bis 1950 fort. Nun sind es rund 73 Prozent. Für die Wirtschafts- und Rechtswissenschaften lag der Anteil in der NS-Zeit relativ hoch. Mit 17,4 Prozent auf das Jahr 1940 gerechnet war er der höchste seit 1887 und wurde auch erst nach 1966 wieder überschritten. Vergleicht man diesen Wert für die NS-Zeit nach Jahren mit der Zeit nach dem Zweiten Weltkrieg, dann ist letzterer bei weitem nicht halb so hoch wie in der NS-Zeit. Erst kurz vor der zweiten Generation in SBZ und DDR, also um den VI. Parteitag der SED im Jahr 1963 und noch vor der 3. Hochschulreform, wuchs ihr Anteil darüber hinaus. Auf der Grundlage der hier vorliegenden quantitativen Daten für die Bergakademie kann für diese Zeit eine umfas-

genannten Zeitraum übereinstimmen, marginalisiert werden. Hingegen ist es nicht möglich, die großen Abweichungen zu Wagenbreth u. a., Technische Universität Bergakademie Freiberg (wie Anm. 12), S. 68, für die "Zahlen der Professoren“ in SBZ und DDR zu diskutieren, da die dortigen Quellen nicht nachvollzogen werden können. Auch fehlt dort eine kritische Abgrenzung zum älteren Forschungsstand zu SBZ und DDR bei Baumgärtel und damit eine Erklärung der genannten Abweichungen zu ebd.

41 Für das Habilitationsalter insgesamt an den Universitäten und technikwissenschaftlichen Instituten im Deutschen Kaiserreich, in Weimarer Republik und NS-Zeit vgl. RINGER, Sociography of German Academics (wie Anm. 28), S. 264 f. Die Werte sind zu denen der Bergakademie Freiberg vergleichbar hoch. 
sende Ideologisierung der DDR-Hochschule als Spezifikum im Vergleich zu den vorherigen politischen Staatsformen nicht geltend gemacht werden. Die 3. Hochschulreform bestätigte insofern lediglich eine Tendenz der Ideologisierung der Hochschule, die sich in Freiberg im Übrigen in ihrer Funktion - unbesehen von der jeweiligen politischen Ordnung - weitgehend staatsloyal positionierte. ${ }^{42} 1982$ war der Anteil der Gesellschaftswissenschaftler, einschließlich der Wirtschaftswissenschaftler, an der Gesamtprofessorenschaft am höchsten. Er betrug nunmehr 29,2 Prozent. Vier Jahre zuvor war der akademische Titel ,Dr. phil.' an der Bergakademie wieder eingeführt worden. Bis 1989 fiel der Anteil der Gesellschafts- und Wirtschaftswissenschaftler wieder auf 23,81 Prozent und erreichte um die Zeit der Wiedervereinigung, präziser gesagt 1993, seinen tiefsten Wert, nämlich 7,52 Prozent. In der politischen Umbruchsituation im Zuge der Wiedervereinigung galten diese Wissenschaften als ideologisch besonders kompromittiert. Bis zum Ende des Untersuchungszeitraums stieg der Anteil der Wirtschafts-, Rechts- und historischen Wissenschaften dann wieder auf rund 15 Prozent.

Waren die Professorenzahlen bis 1949 immer noch niedrig, so zeigt doch die hohe Steigerung die Tendenz der gestiegenen Nachfrage nach naturwissenschaftlich-technischem Wissen im Laufe des 20. Jahrhunderts. Diese Zahlen für die Bergakademie Freiberg sind konform zur Entwicklung in Deutschland. ${ }^{43}$ In der DDR und Bundesrepublik potenzierte sich noch einmal deutlich die Zahl der Freiberger Professoren und Studierenden. Nach einem kurzen Einbruch der absoluten Zahlen für die lehrenden Professoren nach dem Ende des Zweiten Weltkriegs beziehungsweise während der sowjetischen Besatzungszeit stiegen die Zahlen seit der Gründung der DDR - von geringen Abweichungen abgesehen - beständig bis zum Jahr der Wiedervereinigung 1990. Im Verhältnis zu Weimarer Republik und NS-Zeit zusammengenommen verdreifachte sich knapp die Zahl der Professoren im Jahresmittel. Wenn für die sächsischen Hochschulen 1952 ingesamt erst „zwei Drittel des Personalstandes zu Kriegsende erreicht werden" konnten, ${ }^{44}$ dann war der Wert für die Bergakademie zu dieser Zeit bereits ausgeglichen. Der Bergakademie, mit ihrer Spezialisierung auf naturwissenschaftlich-technisches Wissen zur Exploration und Verarbeitung von Rohstoffen, kam für die Industrialisierung der DDR früh ein besonderer Stellenwert zu.

Der Anteil promovierter Professoren war im auf das Jahr gemittelten Wert in der SBZ ungefähr so hoch wie in der NS-Zeit, das heißt hier 84,9 Prozent, und stieg für die DDR-Zeit auf 93,8 Prozent. Erst ab Anfang der 1980er-Jahre waren alle bis zum Ende des Untersuchungszeitraums zu besetzenden Professuren mit Promovierten besetzt.

Während in der SBZ der Anteil jener Professoren, die ihre Promotion oder Habilitation an der Bergakademie abgelegt hatten, an der Gesamtprofessorenschaft bei jähr-

42 Vgl. Norman Pohl, Hochschulreform im Zeichen des Klassenkampfes. Zur Geschichte der Bergakademie Freiberg von 1960 bis 1970, in: Ders./Sabine Schleiermacher (Hg.), Medizin, Wissenschaft und Technik in der SBZ und DDR. Organisationsformen, Inhalte, Realitäten (Abhandlungen zur Geschichte der Medizin und der Naturwissenschaften 107), Husum 2009, S. 173-215, hier S. 182 f., der dies auf die einzelnen Freiberger Professoren bezieht.

43 Vgl. Joachim Radkau, Technik in Deutschland. Vom 18. Jahrhundert bis heute, Frankfurt a. M. u. a. 2008, S. 246-252.

44 Michael Parak, Zwischen politischer Säuberung und akademischer Fluktuation. Elitenaustausch an sächsischen Hochschulen 1945-1952, in: Rainer Behring/Mike Schmeitzner (Hg.), Diktaturdurchsetzung in Sachsen. Studien zur Genese der kommunistischen Herrschaft 1945-1952 (Schriften des Hannah-Arendt-Instituts für Totalitarismusforschung 22), Köln/Weimar/Wien 2003, S. 297-324, hier S. 323. 
lich knapp unter 10 Prozent und damit im Vergleich zu den vorangegangenen Zeiten schon am höchsten lag, stieg dieser Wert während der DDR-Zeit tendenziell stark an, und zwar auf den höchsten Prozentsatz für die geschlossene DDR-Gesellschaft Mitte der 1980er-Jahre von über 60 Prozent. Bis zur Wende im Jahr 1989 sank dieser Wert wieder auf rund 55 Prozent, um dann bis zum Jahr 1992 noch seinen Peak für den gesamten Untersuchungszeitraum mit 62,2 Prozent $\mathrm{zu}$ erreichen. ${ }^{45}$ Anschließend fiel dieser Anteil fast kontinuierlich bis 2011 auf ca. 17 Prozent. Die statistischen Ausreißer um die starken politischen Zäsuren, NS-Zeit zur SBZ und DDR zur Bundesrepublik, verweisen für erstere Zäsur auf den Solidarisierungseffekt in Krisen. Der Investition von Sozialkapital in Netzwerken kam hier ein besonderer Stellenwert zu. Der Anteil Professoren, die ihre Habilitation oder Promotion an der Bergakademie Freiberg gemacht hatten, verdoppelte sich zwischen dem letzten Kriegsjahr und dem Gründungsjahr der DDR im Vergleich zu dem vorangegangenen Zeitraum im Nationalsozialismus. Für den zweiten hier benannten politischen Systembruch sieht die Analyse anders aus. Ausgehend von einem bereits hohen Anteil von deutlich über 50 Prozent an der Gesamtprofessorenschaft zur Wendezeit, fällt die nochmalige Steigerung der Professoren, die ihre Promotion oder Habilitation an der Bergakademie gemacht hatten, weniger signifikant aus. Vor allem die Entlassungen im Bereich der Gesellschaftswissenschaften, die im Wesentlichen ihre Qualifikationen an anderen Hochschulen der DDR abgelegt hatten, tragen zu dieser statistischen Verschiebung bei.

Während der Anteil der im Deutschen Kaiserreich an der Bergakademie Freiberg eingesetzten Professoren, die in Sachsen geboren worden waren, an der Gesamtprofessorenschaft zwar schon gesunken war, aber immer noch bei 60 Prozent stand, sank dieser Anteil in der Weimarer Republik deutlich, und zwar auf 20 Prozent, für die NS-Zeit sowie für die SBZ-Zeit lag er dann jeweils etwas über 30 Prozent, ganz ähnlich wie für die Bundesrepublik. Im Verhältnis zu den drei letzten Werten betrug der Anteil für die DDR-Zeit um die 10 Prozent mehr. Die verbleibenden Anteile verteilen sich im Wesentlichen auf Geburtsorte in anderen deutschen Ländern (auf Territorien nach 1815 gruppiert), lediglich Geburtsorte in Gebieten, die zeitweilig unter Österreichisch-Ungarischer Herrschaft standen, fallen beginnend in der NS-Zeit quantitativ ins Gewicht. Hier waren es 21 Prozent der Professoren, die in diesen Gebieten geboren worden waren, in der SBZ nur noch 6, in der DDR 7 und in der Bundesrepublik 3 Prozent. Berufungen von in anderen Ländern Europas, in Asien oder Südamerika geborenen Professoren blieben deutlich die Ausnahme.

Bei Betrachtung der akademischen Lebensverläufe zeigt sich die Attraktivität eines Wechsels nach oder von Freiberg. Es gelang zunächst bis einschließlich 1870 einen Professor, es handelte sich um Friedrich Mohs, der zuvor eine andere Professur besetzt hatte, für die Bergakademie zu gewinnen. Später wechselten er und Carl Friedrich Naumann an eine Universität. Mohs ging nach Wien und Naumann nach Leipzig. Im Deutschen Kaiserreich waren es bereits vier der neuberufenen Professoren, die von Freiberg wechselten. Damit hatte sich ihr prozentualer Anteil im Vergleich zu dem vorherigen Zeitabschnitt nicht ganz verdoppelt. Er betrug jetzt 8,51 Prozent. Während

45 Vgl. hierzu auch die Diskussion der Hausberufungen bei Karin Zachmann, Mobilisierung der Frauen. Technik, Geschlecht und Kalter Krieg in der DDR (Geschichte und Geschlechter 44), Frankfurt a. M. 2004; dort auf der empirischen Grundlage von RaLPH Jessen, Professoren im Sozialismus. Aspekte des Strukturwandels der Hochschullehrer in der Ulbricht-Ära, in: Hartmut Kaelble/Jürgen Kocka/Hartmut Zwahr (Hg.), Sozialgeschichte der DDR, Stuttgart 1994, S. 217-253, hier S. 232-236. Zachmann (S. 95) schreibt: „Ein spezifisches Merkmal der akademischen Sozialisation in der DDR war ein ausgesprochener Lokalismus, mit dem die Hausberufung zum Regelfall wurde.“ 
es in der Weimarer Republik dann bereits über 50 Prozent mehr waren, sank dieser Anteil in der NS-Zeit wieder leicht. Zwei dieser Professoren waren zuvor von anderen Universitäten nach Freiberg gekommen. Ihr Anteil betrug keine 20 Prozent an allen in der NS-Zeit neuberufenen Professoren. Dieser Anteil sank in der DDR, das heißt von 1949 bis 1990, auf rund 3 Prozent und 6,6 Prozent wechselten in dieser Zeit von Freiberg aus an andere Hochschulen. Bis zur Weimarer Republik war die Bergakademie Freiberg eine Endstationsuniversität in den akademischen Lebensverläufen der Professoren. Dies änderte sich in der Weimarer Republik. Wobei hiervon ein Drittel und alle Wechsel in der NS-Zeit der politischen Zäsur 1945 geschuldet sind. Die Ergebnisse für die Bergakademie in der DDR stehen für eine insgesamt geringe Mobilität in den akademischen Lebensverläufen dieser Zeit.

Von einem Austausch der Professoren durch die SMAD hin zu politisch loyalen Eliten kann für die Bergakademie nur im Sinne einer allgemeinen Rahmensetzung ausgegangen werden. ${ }^{46}$ Dieser wurde verzögert realisiert, ${ }^{47}$ zumal eine Reihe von aus der Bergakademie verdrängten Professoren zunächst bei den Sowjetischen Wissenschaftlich-Technischen Büros Beschäftigung fand und nach kurzer Unterbrechung zurückkehrte. ${ }^{48}$ Loyalitätsbekundungen - etwa in Form einer SED-Mitgliedschaft sollten die Professoren erst mit der allmählichen Verstetigung der DDR-Diktatur zum Ausdruck bringen. ${ }^{49}$ Der Anpassungsdruck auf die Natur- und Ingenieurwissenschaftler sowie Mathematiker war in der DDR ohnehin solange geringer, wie es praktisch noch die Möglichkeit von Freizügigkeit gab. Anwendungsorientierte Wissenschaften waren für den wirtschaftlichen Aufbau notwendig und fielen nicht unter den ideologischen Herrschaftsvorbehalt gegenüber geisteswissenschaftlichen Fächern. ${ }^{50}$ Damit war das Gros der Professorenschaft an der Bergakademie bis zur Schließung der DDRGrenze im Jahr 1961 politisch privilegiert.

In der Bundesrepublik nahm die Zahl der lehrenden Professoren - bevor die Bergakademie 1992/93 Technische Universität wurde - kurzzeitig ab, um dann für rund ein Jahrzehnt zwischen 1992 und 2001 im Jahresmittel mit rund 126 Professoren den höchsten Durchschnittswert in ihrer Geschichte zu erreichen. Wuchs die Zahl der Professoren in den ersten zehn vollen Kalenderjahren der DDR (1950-1959) von 22 auf 42, stieg sie in den ersten zehn Kalenderjahren der Bundesrepublik (1991-2000) von 86 auf 128. Kamen im Jahr 1949 auf einen Professor gerade einmal 26 Studenten,

46 Vgl. wie Parak, Elitenaustausch (wie Anm. 44), S. 297, sein Fazit Helga A. Welsh, Revolutionärer Wandel auf Befehl? Entnazifizierungs- und Personalpolitik in Thüringen und Sachsen (1945-1948) (Vierteljahrshefte für Zeitgeschichte 58), München 1989 gegenüber stellt; ebd., S. 324: „Die personelle Umgestaltung der Hochschulen nach den Gesichtspunkten der SED-Diktatur konnte erst in den Fünfziger- und Sechzigerjahren erreicht werden. Entscheidend waren eine langfristige Nachwuchsplanung und die Schließung der offenen Westgrenze mit dem Mauerbau 1961.“ Parak formuliert damit auch eine Gegenthese zum Titel des Sammelbandes, spricht dieser doch von Diktaturdurchsetzung explizit für die Zeit unmittelbar nach der NS-Diktatur, das heißt nach 1945 und bis 1952.

47 Für die verzögerte Umsetzung der Entlassung aller ehemaligen NSDAP-Mitglieder vgl. PARAK, Elitenaustausch (wie Anm. 44), S. 303, Anm. 25.

48 Vgl. ebd., S. 320 f.; und Alexander Haritonow, Entnazifizierung an der Bergakademie Freiberg 1945-1948, in: Bildung und Erziehung 45 (1992), H. 4, S. 433-436, hier S. 434.

49 Siehe weiter unten.

50 Vgl. Ralph Jessen, Akademische Elite und kommunistische Diktatur. Die ostdeutsche Hochschullehrerschaft in der Ulbricht-Ära, Göttingen 1999, S. 279 f. und 285; sowie Ders., Professoren im Sozialismus (wie Anm. 45), S. 225. 
so waren es 1956 schon 96. Nach diesem kontinuierlichen Anstieg sank das Verhältnis Professor zu Studenten leicht. 1963 betrug dieser Wert gerundet 1:71.51

Die erstmalige Besetzung einer Professur mit einer Frau - 1978 erhielt Maja Krumnacker ${ }^{52}$ die außerordentliche Professur für Metallkunde - muss bis zum Ende der DDR-Zeit für die Bergakademie noch als Ausnahme angesehen werden. Unter den ab 1990 berufenen 210 Professoren befanden sich dann 12 Frauen. Der Anteil Frauen auf Lehrstühlen blieb also auch an der Bergakademie in der Bundesrepublik bis 2015 gering, das heißt knapp unter 6 Prozent bei den Neuberufungen. Auf die zugrunde gelegten Fachbereiche verteilen sich die mit Frauen besetzten Professuren wie folgt: Fachbereich Mathematik/Naturwissenschaften 4, Fachbereich Technikwissenschaften 5 und Fachbereich Wirtschafts- und Geisteswissenschaften 3.

Betrachtet man die Entwicklung der Fachbereiche allgemein, dann lag der Peak des höchsten Anteils an Ingenieurwissenschaftlern seit 1961 (59,57 Prozent) im Jahr 1993 mit 57,14 Prozent am höchsten. 1993 waren 133 Professoren an der TU Bergakademie tätig, das war die höchste je erreichte absolute Zahl. In den folgenden Jahren fiel der Anteil Ingenieurwissenschaftler an der Gesamtprofessorenschaft bis 2009 um über 15 Prozent. Ab dieser Zeit glichen sich bis zum Ende des Untersuchungszeitraums der Fachbereich Mathematik/Naturwissenschaften und der Fachbereich Technikwissenschaften wieder in der Professorenstärke an.

51 Baumgärtel, Geschichte der Bergakademie (wie Anm. 12), S. 61, zeigt den starken Anstieg des wissenschaftlichen Mittelbaus ab 1952 bis 1960, der für das fachliche Betreuungsverhältnis der Studierenden über obige Angaben hinaus mit in Rechnung zu stellen ist. Die geringe Abweichung um zwei Professorenstellen zur Zahl hier für das gesamte Jahr 1992 resultiert aus dem genauen Stichdatum 7. Oktober 1992 bei Franco Lehmann, Zwischen Selbstbestimmung und Fremdsteuerung. Die Bergakademie Freiberg im Transformationsprozess 1989 bis 1993, Diss. TU Bergakademie Freiberg 2013, S. 224, mit Bezug auf das Protokoll der 54. Senatssitzung der Bergakademie Freiberg am 6. Oktober 1992. Die Abweichungen zur nicht publizierten Zusammenstellung von Roland Volkmer, ebd., S. 249 zitiert, für September 1989, Februar 1990 und September 1990 können hier nicht interpretiert werden. Vgl. für die absolute Zahl der pro Jahr Studierenden für die Zeit 1946 bis 1963 Johannes WeIssflog/Eberhard WäCHTLER, Die Freiberger Studentenschaft seit 1946, in: Bergakademie Freiberg (wie Anm. 2), Bd. 1, S. 390-403, hier S. 399.

52 Die Feststellung von Zachmann, Mobilisierung der Frauen (wie Anm. 45), S. 104, Anm. 241, dass Maja Krumnacker schon 14 Jahre zuvor die „üblichen Berufungsvoraussetzungen“ erfüllt hätte, da ,in den Ingenieurwissenschaften [...] der Anteil habilitierter Kader gering“ war, kann hier mit Zahlen für die Bergakademie kritisch unterlegt werden. 1978 betrug der Anteil Habilitierter an der Gesamtprofessorenschaft 73 Prozent und in den zehn vorangegangenen Jahren (1969-1978) im Durchschnitt 68,8 Prozent. Diese Steigerung des Anteils setzte sich zunächst bis zum Ende der DDR fort (1989= 85 Prozent). Professuren von Habilitierten bzw. B-Promovierten überwogen an der Bergakademie also deutlich in dem Jahrzehnt, in dem Krumnacker ihre Professur antrat. Damit muss, anders als bei Zachmann, die Habilitation zu den üblichen Voraussetzungen einer Professur gerechnet werden. Eine Benachteiligung Krumnackers kann auf dieser Grundlage nur schwerlich geltend gemacht werden. Zur Benachteiligung von Frauen bei der Vergabe von Professuren an der Bergakademie siehe oben. 


\section{Politik, Wissenschaft und Wirtschaft als interdependentes Movens für die akademischen Lebensverläufe der Freiberger Professorenschaft}

Wenn für wissenschaftlich-technische Eliten gilt, dass sie sowohl im Feld der Wissenschaft als auch im Feld der Wirtschaft und im Feld der Politik aussichtsreich positioniert sind, ${ }^{53}$ dann lässt sich dies zum Beispiel am Wechsel im Lebensverlauf der Professoren aus der Wirtschaft in die Wissenschaft und umgekehrt ablesen. Für die Bergakademie waren diese Positionierungen in Wirtschaft und Wissenschaft zumindest bis zum Ende der DDR typisch. Stark war diese Verbindung schon in der Geschichte der Bergakademie vor 1869, als die Bergakademie Freiberg noch in die Verwaltung des sächsischen Bergstaates eingebunden war. ${ }^{54}$ Im Deutschen Kaiserreich arbeiteten noch rund zwei Drittel der Freiberger Professoren in ihren Karrieren auch in der Wirtschaft. In der Weimarer Republik stieg dieser Wert auf vier Fünftel und lag damit sogar über dem Wert für die ersten 100 Jahre. Von den in der NS-Zeit berufenen Professoren arbeitete immer noch die Hälfte im Laufe ihres Berufslebens in der Wirtschaft und für die Gründungsgeneration der DDR schnellt dieser Wert sogar wieder auf vier Fünftel hoch, um danach allmählich abzufallen. Generell kann der Bergakademie so eine hohe Verbindung von Theorie und Praxis attestiert werden.

$\mathrm{Zu}$ einer konventionellen Orientierung auf das Feld der Politik, das heißt auf Herrschaft, gehörten für die überwiegend männliche Professorenschaft der Militärdienst oder auch dessen ideologische Flankierung. Über ein Viertel der im Deutschen Kaiserreich berufenen Freiberger Professoren unterzeichnete die Erklärung der Hochschullehrer des Deutschen Reichs zum Ersten Weltkrieg. Zwischen 1900 und 1933 wurde die Bergakademie über reichlich drei Viertel der Jahre eben auch von Unterzeichnern dieser militaristischen Erklärung geführt. Hierzu gehörten Rektoren wie Erwin Papperitz, Richard Beck, Friedrich Kolbeck, Johannes Galli, Carl Schiffner, Erich Wandhoff, Otto Fritzsche und Reinhold Freiherr von Walther. Letztere vier waren in der Weimarer Republik Rektoren, Papperitz und Kolbeck ebenso, aber jene waren dies zuvor auch noch im Deutschen Kaiserreich. Von Walther wurde im Jahr der Macht-

53 So wäre dem wissenschaftstheoretischen Optimismus von Luhmann über die Geschlossenheit einzelner Systeme der Gesellschaft, wie dem der Wissenschaft, angesichts obiger Befunde eine erhebliche interpretative Aufgabe beiseitezustellen. Etwa in der Hinsicht, wie Niklas Luhmann, Die Wissenschaft der Gesellschaft, Frankfurt a. M. 1992, S. 304, selbst die Grenzen der Interpretation deutlich macht, wenn er schreibt, „daß die Umwelt kausal auf das System einwirken kann; oder genauer: daß ein Beobachter sehen kann, daß die Umwelt auf das System einwirkt.“ Die obigen Befunde aus den angewandten Wissenschaften verweisen jedenfalls stark auf die Wechselwirkungen zwischen den drei genannten Feldern und lassen Skepsis gegenüber einer Geschlossenheit der Systeme angezeigt sein. Zu einer ähnlichen Kritik vgl. Rudolf STichweH, Wissenschaft, Universität, Professionen. Soziologische Analysen, Bielefeld 2013, S. 109.

54 Vgl. weiter oben und für die Gründungsphase der Bergakademie wie WolfHard Weber, Innovationen im frühindustriellen deutschen Bergbau und Hüttenwesen. Friedrich Anton von Heynitz, Göttingen 1976, S. 151, aufzeigt, in welcher Art und Weise Friedrich Anton von Heynitz als ein entscheidender Protagonist der Reformen des sächsischen Bergstaates, der die Gründung der Bergakademie mitvorantrieb, $\mathrm{Zu}$ gang zum sächsischen Hof hatte. Heynitz steht denn auch am Anfang von Radkaus Geschichte der „Technik in Deutschland vom 18. Jahrhundert bis heute“, und zwar mit einer Audienz beim preußischen König, unter dem er inzwischen das „Berg- und Kriegsdepartement" führte; RADKAU, Technik in Deutschland (wie Anm. 43), S. 10; zur Bedeutung des Bergbaus für die Entwicklung der Technik und für die Verwissenschaftlichung des Bergbaus vgl. aber auch ebd., S. $115 \mathrm{f}$. 
übernahme durch die Nationalsozialisten Prorektor - nachdem er zuvor Rektor gewesen war - und trat der NSDAP bei.55 Schiffner, Fritzsche, Wandhoff, Kolbeck und Papperitz unterzeichneten gleich 1933 auch noch das Bekenntnis der Professoren an den deutschen Universitäten und Hochschulen zu Adolf Hitler und dem nationalsozialistischen Staat und standen damit Georg Brion, Franz Kögler und Otto Brunck, die in der Weimarer Republik Rektoren gewesen waren, nicht nach. Die Rektoren der NS-Zeit hatten alle dieses Bekenntnis unterzeichnet, von der seinerzeitigen Professorenschaft insgesamt 86 Prozent. Die Zustimmung zum politisch-kulturellen Mainstream, ${ }^{56}$ die Orientierung auf Herrschaft, war für die Professorenschaft selbstverständlich und für Gesellschaftsordnungen mit hohem politischen Normierungsdruck etwa an einem solchen Bekenntnis oder an der Zugehörigkeit zur Staatspartei und ihren Organisationen klar abzulesen. Dies gilt retrospektiv auch für jene, deren Investitionen in eine aussichtsreiche Positionierung im Feld der Macht sich als Fehlinvestitionen erwiesen, wie bei den ehemaligen NSDAP-Mitgliedern in der bergakademischen Professorenschaft der jungen DDR. Während in der gesamten DDR 1954 28,4 Prozent der Hochschullehrer ehemalige NSDAP-Mitglieder waren und ihr Anteil bei den technischen Disziplinen 41,9 Prozent, bei den Wirtschafts-, Gesellschafts- und Rechtswissenschaften 16,6 Prozent ${ }^{57}$ und bei den Naturwissenschaften 31,2 Prozent betrug, ${ }^{58}$ lag der Anteil ehemaliger NSDAP-Mitglieder an der Bergakademie Freiberg an der gesamten Professorenschaft im selben Jahr bei 34,4 Prozent, also 6 Prozentpunkte über dem Wert für die gesamte DDR. Nach Fachbereichen an der Bergakademie Freiberg aufgeschlüsselt waren dies 33,3 Prozent ehemalige NSDAP-Mitglieder bei den technischen Disziplinen und 38,5 Prozent bei den Naturwissenschaftlern und Mathematikern. Bei den Wirtschafts-, Gesellschafts- und Rechtswissenschaften betrug ihr Anteil null. Letzteres lag am zunächst radikalen Austausch der Professoren in diesen Disziplinen aufgrund des politischen Systembruchs nach 1945 bzw. 1949. Diese Zahlen veranschaulichen, dass diese Disziplinen in politischen Umbruchsituationen, vergleiche hierzu auch die stark sinkenden Professuren der Wirtschafts-, Gesell-

55 Wenn Norman Pohl, Die Bergakademie Freiberg im Nationalsozialismus. Ein Werkstattbericht, in: Günther Heydemann/Jan Erik Schulte/Francesca Weil (Hg.), Sachsen und der Nationalsozialismus (Schriften des Hannah-Arendt-Instituts für Totalitarismusforschung 53), Göttingen 2014, S. 251-266, hier S. 252, schreibt: „auf der Ebene der Ordinarien [ist] noch zu Beginn des Jahres 1933 kein NSDAP-Mitglied zu verzeichnen“, dann zeigt obiges Beispiel des Rektors beziehungsweise dann Prorektors Reinhold Freiherr von Walther, wie NSDAP-Mitgliedschaften schon mit dem Jahr 1933, dies gilt auch für die Professoren Walter Hoffmann und Johannes Steinbrecher, zum politischen Bekenntnis der Freiberger Professorenschaft dazuzugehören begannen; vgl. die entsprechenden Biografien von Hartmut Schleiff in Schleiff/Volkmer/Kaden, Catalogus Professorum Fribergensis (wie Anm. 1), S. 114, 123, 129. Albrecht, Bergakademie Freiberg (wie Anm. 29), S. 203, stellt namentlich in diese Reihe auch noch Prof. Dr. Hellmut von Philipsborn sowie die Dozenten Kurt Säuberlich und Robert Höltje. Letztere erhielten später in der NS-Zeit eine Professur an der Bergakademie Freiberg.

56 Für die hier vorgetragenen Zahlen vgl. die entsprechenden Einzelbiografien in Schleiff/Volkmer/Kaden, Catalogus Professorum Fribergensis (wie Anm. 1). Darüber hinaus ist dies auch symptomatisch an den Jubiläumsfeiern der Bergakademie im Nationalsozialismus abzulesen; vgl. Albrecht, Bergakademie Freiberg (wie Anm. 29), S. 192-195.

57 Vgl. Jessen, Professoren im Sozialismus (wie Anm. 45), S. 226.

58 Vgl. Mitchell G. Ash, Verordnete Umbrüche - Konstruierte Kontinuitäten. Zur Entnazifizierung von Wissenschaftlern und Wissenschaften nach 1945, in: Zeitschrift für Geschichtswissenschaft 43 (1995), S. 903-922, hier S. 913. 
schafts-, Geschichts- und Rechtswissenschaften im Zuge der Wiedervereinigung, unter dem besonderen Vorbehalt ethisch-ideologischer Kontaminierung durch den jeweils vorangegangenen Staat standen, insbesondere dann, wenn diese vorangegangenen Staaten Diktaturen mit hohem politischen Normierungsdruck waren. Aber schon im Jahr 1956 berief man beispielsweise das ehemalige NSDAP-Mitglied Günther Hollweg, er war 1945 in die CDU eingetreten, als Professor für Organisation des Bergbaubetriebes an die Bergakademie Freiberg und ernannte ihn zugleich zum Direktor des Instituts für Ökonomie der Montanindustrie. Von 1957 bis 1961, in letzterem Jahr wurde er Rektor der Bergakademie Freiberg, war er Dekan der Fakultät für Ingenieurökonomie. Sein Rektorat übte er bis 1963, dem Jahr seines Todes, aus. Seine fachliche Kompetenz vor allem im Bereich der Braunkohlenförderung ließ ihn in der DDR trotz CDU- und vorangegangener NSDAP-Mitgliedschaft auch nach Schließung der DDRGrenze weiterhin Karriere machen. Er war bereits der vierte Rektor der Bergakademie seit 1955 in Folge, der in der NS-Zeit NSDAP-Mitglied gewesen war. Hollwegs direkter Vorgänger, Oscar Oelsner, trat 1947 in die SED ein. Diese biografische Kombination der NSDAP- und späteren SED-Mitgliedschaft traf auch noch auf einen der Nachfolger Hollwegs im Rektorenamt, namentlich auf Karl-Friedrich Lüdemann (Amtszeit 1965-1967), zu. Von den ehemaligen NSDAP-Mitgliedern unter den Freiberger Professoren zur DDR-Zeit trat eine ganze Reihe der neuen Staatspartei SED bei und zeigte damit Elitenkonformität. ${ }^{59}$ In der DDR war das äußere Zeichen einer hinreichenden Systemkonformität die SED-Mitgliedschaft, hingegen können diese Zahlen über tiefer gehende persönliche Anschauungen und politische Motivationen der Professoren nichts sagen. Der Anteil an SED-Mitgliedern unter der Professorenschaft der Bergakademie lag 1950 bei 26 Prozent ${ }^{60}$ und entsprach damit ungefähr dem Niveau bei den sächsischen Hochschullehrern. ${ }^{61}$ In den letzten Jahren der DDR-Diktatur lag dieser Wert für die Bergakademie Freiberg gar bei 88 Prozent $^{62}$ und damit ca. 8 Pro-

59 Vgl. das Begriffsangebot von Ash, ebd., S. 904: „multivalent“. Für entsprechende Zahlen vgl. weiter unten. Wenn man in der Forschungsliteratur auf einzelne Fälle einer NSDAP- und anschließenden SED-Mitgliedschaft von Professoren der Bergakademie Freiberg rekuriert, wird auch Kurt Säuberlich genannt, so ILKo-SASCHA KowalCzuK, Geist im Dienste der Macht. Hochschulpolitik in der SBZ/DDR 1945 bis 1961, Berlin 2003, S. 359; oder PoHL, Hochschulreform (wie Anm. 42), S. 183. Hingegen geht aus der „Aufstellung der Personalien für das Abgeordnetenarchiv der Volkskammer“ im Bundesarchiv, Sign. DA1/1817, Bl. 82, hervor, dass Säuberlich zu diesem Zeitpunkt (Ende der 1950er-Jahre) „parteilos“ war. Auch die aktuelle Auskunft durch Kowalczuk anhand der Unterlagen der Behörde des Bundesbeauftragten für die Stasi-Unterlagen (BStU), MfS, AIM 18744/64 (2 Bände) zeigt, dass Säuberlich kein Mitglied der SED war. Daher ist er nicht in obiger Zählung enthalten; vgl. so auch Hartmut Schleiff in Schleiff/Volkmer/Kaden, Catalogus Professorum Fribergensis (wie Anm. 1), S. 156. Vgl. für die oben genannten Rektoren Prof. Dr.-Ing. habil. Oscar Walter Oelsner, Prof. Dr.-Ing. Karl-Friedrich Lüdemann und Prof. Dr.-Ing. Günther Max Ludwig Hollweg die ent-sprechenden Kurzbiografien; ebd., S. 209, 211, 214.

60 Otfried Wagenbreth/Werner Arnold, Studium an der Bergakademie Freiberg 1946-1950, in: Zeitschrift für Freunde und Förderer der Technischen Universität Bergakademie Freiberg 3 (1994/1996), H. 1/2, S. 16-23, hier S. 22.

61 Vgl. ParaK, Elitenaustausch (wie Anm. 44), S. 324.

62 Vgl. Wagenbreth/Arnold, Studium an der Bergakademie (wie Anm. 60), S. 22. Für ähnliche Ergebnisse zur SED-Mitgliedschaft an der Bergakademie Freiberg im Vergleich zu allen Hochschulen der DDR für 1983/84 vgl. LeHmann, Zwischen Selbstbestimmung und Fremdsteuerung (wie Anm. 51), S. 48; ebenso Bertram Triebel, Die Partei und die Hochschule. Eine Geschichte der SED an der Bergakademie Freiberg 
zent über dem Durchschnittswert für die Professoren in der DDR insgesamt. ${ }^{63}$ Der Anteil der SED-Mitglieder an den überachtzehnjährigen Einwohnern der DDR betrug in den 80er-Jahren 17,4 Prozent. ${ }^{64}$ Eine SED-Mitgliedschaft als beförderndes Moment akademischer Karrieren gewann in der DDR spätestens ab der zweiten Generation zunehmend an Bedeutung. Die akademische Elite war also deutlich stärker in die SEDDiktatur eingebunden als die Bevölkerung generell. Bei diesen Zahlen der SED-Mitgliedschaft für die späte DDR muss in Rechnung gestellt werden, dass die extremen Sozialaufsteiger erst in den 1950er-Jahren als Arbeiter- und Bauernkinder massiv gefördert worden sind. Von ihnen studierten 1960 rund 60 Prozent auf die Gesamtstudierendenschaft der Bergakademie gerechnet. ${ }^{65}$ Lag die Bergakademie mit dem Anteil Studierender der Arbeiter- und Bauernkinder noch bis einschließlich 1950 über dem DDR-Durchschnitt, ${ }^{66}$ hatte sie sich 1960 in den DDR-Trend eingefügt. ${ }^{67}$ Diese Sozialaufsteiger galten ihre Loyalität auch in SED-Mitgliedschaft ab und rückten bei der hohen Selbstrekrutierungsrate, das heißt an Berufungen von an der Bergakademie wissenschaftlich Sozialisierten und Qualifizierten, allmählich nach. ${ }^{68}$ Auch aus diesem Grund nahm der Anteil an SED-Mitgliedern unter den Professoren mit der Dauer der Diktatur zu, ${ }^{69}$ hielt doch die Diktatur der SED zwei Generationen, die NS-Diktatur zuvor nur wenig mehr als eine halbe. Dennoch hatte in letzterer der politische Normierungsdruck bereits nach nur fünf Jahren NS-Herrschaft zu einer Mehrheit an NSDAP-Mitgliedschaften unter der Freiberger Professorenschaft geführt. ${ }^{70}$ Auch

(Freiberger Forschungen zur Wissenschafts- und Technikgeschichte 1), Leipzig 2015, S. 138. Wie Wagenbreth und Arnold für 1989 weist Triebel, ebd., S. 255, auch für 1984 88 Prozent SED-Mitglieder unter den Professoren der Bergakademie Freiberg nach.

63 Vgl. Hans-Ulrich Wehler, Deutsche Gesellschaftsgeschichte, Bd. 5: Von der Gründung der beiden deutschen Staaten bis zur Vereinigung, München 2008, S. 225.

64 Vgl. ebd., S. 324: „In den 80er Jahren hatte die SED-Diktatur das Bild einer lückenlosen Allgegenwart vermittelt. 17,4 \% der erwachsenen Bevölkerung gehörten der Einheitspartei an, 8,8 Millionen dem von ihr gesteuerten FDGB, 2,3 Millionen der FDJ und 1,5 Millionen den Jungen Pionieren." Vgl. auch Andreas Malycha/Peter Jochen WinTERS, Die SED. Geschichte einer deutschen Partei, München 2009, S. 416. Dort steht für 1989 ,2.296.775“ SED-Mitglieder. Auf 12700620 über achtzehnjährige Einwohner im Jahr 1989 (Angabe aus Gabriele Franzmann, Bevölkerung in der ehemaligen DDR 1946-1989, GESIS Datenarchiv, Köln. ZA8267 Datenfile Version 1.0.0, doi: 10.4232/ $1.8267,2009)$ gerechnet, ergeben sich davon 18,08 Prozent SED-Mitglieder.

65 Vgl. Weissflog/Wächtler, Freiberger Studentenschaft (wie Anm. 51), S. 398.

66 Vgl. ebd., S. 393.

67 Vgl. Helmuth Albrecht/Norman Fuchsloch, Die Bergakademie Freiberg im Kontext der Hochschulgeschichte der SBZ/DDR (1945-1989), in: Technische Universität Bergakademie. Festgabe zum 300. Jahrestag (wie Anm. 2), S. 13-47, hier S. 14.

68 Siehe weiter oben.

69 Vgl. insbesondere zum Forschungsstand für den Schichtenrekrutierungs- und SEDMitgliedschaftsanteil bei allen Professoren in der DDR AlbRecht/FuchsLoch, Bergakademie Freiberg (wie Anm. 67), S. 15.

70 Vgl. PoHL, Bergakademie Freiberg im Nationalsozialismus (wie Anm. 55), S. 252: „dass auf der Ebene der Ordinarien noch zu Beginn des Jahres 1933 kein NSDAP-Mitglied zu verzeichnen war. Das Zusammenwirken einer gezielten Berufungspolitik sowie die Aufhebung des Aufnahmestopps in die NSDAP 1937 führten aber dazu, dass bereits ab 1937 Nationalsozialisten an der Bergakademie unter den Professoren in der Mehrheit waren." Vgl. für weitere Technische Hochschulen, wie die in Hannover, Berlin und Braunschweig, in denen nach knapp einer halben Generation seit Beginn der NS-Diktatur über 50 Prozent NSDAP-Mitglieder unter den Professoren lehrten, den Vortrag 
nach zehn Jahren von 1949 an gerechnet wurde dieser Anteil an SED-Mitgliedschaften in der DDR nicht erreicht. Er lag 1958 bei 48,4 Prozent. Davon waren 35,7 Prozent ehemalige NSDAP-Mitglieder. 1968, also am Ende der ersten Generation der DDR, lag der Anteil an SED-Mitgliedern an der Freiberger Professorenschaft bei 40,2 Prozent. Davon waren trotz des inzwischen großen zeitlichen Abstands immer noch 6,2 Prozent ehemalige NSDAP-Mitglieder. Erst später werden, wie bereits erwähnt, die SED-Mitglieder die Mehrheit unter den Professoren der Bergakademie stellen.

\section{Die Bergakademie Freiberg als naturwissenschaftlich-technische Hochschule in der Dynamik der modernen Gesellschaft: Fazit}

Wenn Ende des 19. Jahrhunderts von den Technischen Hochschulen als Kinder der Zeit gesprochen und so eine Abgrenzung zu den Universitäten gesucht wurde, ${ }^{71}$ dann verstand es sich nicht von selbst, dass jede Technische Hochschule gleichermaßen am jungen Erfolg dieses Hochschultypus teilhaben konnte. Die neu institutionalisierte naturwissenschaftlich-technische Bildung stand in einem wechselseitig befördernden Verhältnis zur Industrialisierung, zunächst über die Mechanisierung und später auch die Automatisierung mit all ihren Facetten. Für die Bergakademie Freiberg galt es, sich für diese Anforderungen - zum Beispiel mit den Zeunerschen Reformen zu Beginn des Deutschen Kaiserreichs - neu aufzustellen: Dies kommt unter anderem in den steigenden Zahlen für die Professorenschaft (Abb. 2), den neu eingerichteten Fächern und der allmählichen Einbindung in die standardisierten Qualifikationsabschlüsse, die nunmehr überall im deutschen Staat galten und damit eine größere Reichweite hatten, zum Ausdruck. Die gestiegene Nachfrage nach naturwissenschaftlich-technischem Wissen bei der Steigerung des Wirkungsgrades, der ökonomischen Effizienz von technischen Instrumenten, Maschinen und Technologien spiegelt sich in den tendenziell gestiegenen Zahlen der Professorenschaft, aber auch in den Zahlen der Studierenden. Leichte Schwankungen sind hier diskutiert worden, ${ }^{72}$ die grundsätzliche Entwicklung, vor allem die hinzugewonnene hohe Dynamik ab dem Ende des Zweiten Weltkriegs zeigt jedoch für die Bergakademie Freiberg ein klares Ergebnis (Abb. 2).

Zwar kann die Geschichte einer die Industrie befördernden naturwissenschaftlichtechnischen Bildung und Forschung mit ihren wechselnden Wertbestimmungen nicht linear als Fortschritt erzählt werden, die Entwicklung der Qualität und Quantität von Personal verweist aber darauf, dass die naturwissenschaftlich-technische Elite immer wieder entscheidend für Problemlösungen ist, nicht zuletzt auch für Lösungen von

von Michael Jung zur Konferenz „Technische Hochschulen in der Zeit des Nationalsozialismus" vom Mai 2015; vorgestellt in Michael Jung/Michele Barricelli, Technische Hochschulen in der Zeit des Nationalsozialismus, in: Jahrbuch für Universitätsgeschichte 18 (2015), S. 261-269, hier S. 263.

71 Vgl. wie Albrecht, Technische Bildung (wie Anm. 36), S. 25, im Anschluss an Egon Zöllers Urteil von 1891 die Technischen Hochschulen als Kinder der Zeit bezeichnet, ohne dabei deren Vorgeschichte auszublenden. Auch für die Bergakademie und die Technische Hochschule Dresden entsteht gegen Ende des Jahrhunderts eine neue Dynamik der Entwicklung, die zum Beispiel aus dem Polytechnikum die Technische Hochschule Dresden formt.

72 Die Fluktuationsquote (Ausgeschiedene/Gesamtzahl*100\%) beträgt gemittelt bis zum Kaiserreich 3,1 Prozent, für die Bergakademie im Kaiserreich 5,9 Prozent, in der Weimarer Republik 3,5 Prozent, in der NS-Zeit 5 Prozent, in der DDR 4,8 Prozent und in der Bundesrepublik bis 20147 Prozent. 
Problemen, die sie zuvor mitgeschaffen hat, wie etwa den Mangel an Energieträgern, zum Beispiel Holz und später Kohle. ${ }^{73}$ Die Zunahme des Gewichts von Natur- und Technikwissenschaften - einschließlich der Mathematik - ist so zum Ausweis der Entwicklung moderner Gesellschaften geworden.

73 Wenn Ortwin Renn, Technikfolgenabschätzung. Aufgaben, Methoden, Aussichten, in: Heinz Kunle/Stefan Fuchs (Hg.), Die Technische Universität an der Schwelle zum 21. Jahrhundert. Festschrift zum 175jährigen Jubiläum der Universität Karlsruhe (TH), Berlin/Heidelberg 2000, S. 419-429, hier S. 421, der ethisch abstrakten Normativität Hans Jonas' zu einem Technikvorbehalt die ebenso abstrakte Skepsis der prospektiven Technikfolgenabschätzung entgegensetzt, zeigt sich die Chance, die in der Umweltgeschichte liegt. Sie kann retrospektiv Handlungsmuster, Ressourceneinsatz und -verbrauch tatsächlich in den Blick nehmen. 